Why motor imagery isn't really motoric: Towards a reconceptualization in terms of effect-based action control.

Patric Bach ${ }^{1}$, Cornelia Frank ${ }^{2}$, Wilfried Kunde ${ }^{3}$

${ }^{1}$ School of Psychology, University of Aberdeen, William Guild Building, Kings College, Aberdeen, United Kingdom.

${ }^{2}$ Department of Sports and Movement Science, School of Educational and Cultural Studies, Osnabrück University, Osnabrück, Germany

${ }^{3}$ Department of Psychology, Julius-Maximilians-Universität Würzburg, Röntgenring 11, Würzburg, Germany

Corresponding author: patric.bach@abdn.ac.uk

Running head: Motor or Effect Imagery?

Keywords: imagery, motor imagery, action planning, action control, intentional action, forward models, inverse models 


\begin{abstract}
Overt and imagined action seem inextricably linked. Both follow similar timings, activate shared brain circuits, and motor imagery influences overt action and vice versa. Motor imagery is therefore often assumed to rely on the motor processes governing action execution itself, which allow one to play through or simulate actions offline. Here, we advance a very different conceptualization. In this view, the links between imagery and overt action do not arise because action imagery is intrinsically motoric, but because action planning is intrinsically imaginistic and occurs in terms of the perceptual effects we want to achieve. Viewed like this, the term 'motor imagery' is a misnomer of what is more appropriately portrayed as 'effect imagery'. In this article, we review the evidence for imagery-execution overlaps through this new lens and argue that they indeed emerge because every action we execute is planned, initiated and controlled through an imagery-like process. We highlight findings that this new view can now explain and point out open questions.
\end{abstract}




\section{Why motor imagery is not really motoric: Towards a reconceptualization in terms of effect-based action control.}

Overt and imagined action seems inextricably linked. Before undertaking a difficult motor task, people often experience themselves imaging what they intend to do, and the form this imagination takes (e.g., imagining intended outcomes or motor behaviours) affects their success and subsequent learning (e.g., Land, Frank, \& Schack, 2014; Woolfolk et al., 1985ab). Sometimes, people even imagine behaviours they will execute at a much later time and in a different environment, for example, when they mentally play through the actions of their sport from the privacy of their home. Again, this form of motor imagery - sometimes termed mental practice, mental training or motor imagery training (Schack, Essig, Frank, \& Koester, 2014; for definitions and conceptualizations, see Morris, Spittle, \& Watt, 2005) directly affects later performance (for meta-analysis, Driskell, Copper, \& Moran, 1994; Simonsmeier, Andronie, Buecker, \& Frank, 2020; Toth, McNeill, Hayes, Moran, \& Campbell, 2020) and is recommended by most coaches of professional athletes (Mayer \& Hermann, 2019). Purely mental practice can even increase measured muscle strength, from simple finger contractions to leg pressing and triceps extension, albeit not to the same extent as actual physical practice (Yue \& Cole, 1992, for recent replications and review see, Paravlik, Slimani, Tod, Marusic, Milanovic \& Pisot, 2018; Reiser, Büsch, \& Munzert, 2011; Smith, Collins, \& Holmes, 2003).

Studies from experimental psychology and cognitive neuroscience also support this coupling of overt and imagined action. There are tight correspondences between the timing of imagined and overt actions (Decety, Jeannerod, Prablanc, 1989; Wohlschläger \& Wohlschläger, 1998; for a critical review, see Guillot \& Collet, 2005), between the activated brain structures in parietal and premotor cortices (for reviews, see Lotze \& Halsband, 2006; 
Hétu et al., 2013; O’Shea \& Moran, 2017), and between the lawful regularities that govern the kinematics of both overt and imagined action (e.g., Fitt's law, Decety \& Jeannerod, 1995; two-thirds power law, Karklinsky \& Flash, 2015; Papaxanthis, Paizis, White, Pozzo \& Stucchi, 2012). Moreover, several studies show that motor imagery can engender (subthreshold) activation in the muscles used in the imagined behaviour (Jacobson, 1931; 1932; Munzert \& Krüger 2018; Lutz, 2003; Guillot et al., 2007; Guillot, Lebon, Rouffet, 2010), and, conversely, that executing motor actions makes imagining the same actions easier and imagining different actions harder (e.g., Wohlschläger, 1996; 2001; Callow et al., 2006; Guillot, Moschberger \& Collet, 2013; for a broader review of the effects of such "dynamic motor imagery", see Guillot, under review). The link from imagined to overt behaviour is so strong that it provides the basis for several (stage) magical phenomena. In Chevreul's pendulum and the Ouija board, for example, seemingly supernatural motions happen simply because participants' imagined motions are, unbeknownst to them, translated into subliminal hand and finger movements that are made visible by the devices (Chevreul, 1833; Easton \& Shor, 1975, 1976, 1977).

A standard explanation for these findings is that imagery of action is an intrinsically motoric process. This view assumes that motor imagery, in a form of neural reuse (e.g., Anderson, 2010), draws upon the same neuronal networks and cognitive processes that underlie action execution itself (Jeannerod, 1994; Jeannerod \& Decety, 1995). As a potential mechanism, it has been proposed that the brain predicts - via forward models - the sensory consequences that each of its motor commands will produce, so that it can anticipate the visual, tactile, and proprioceptive sensations that will soon be registered (e.g., Miall \& Wolpert, 1996; Sperry, 1950). During overt action, such predictions may allow the actor to filter out predicted sensations (e.g., Reichenbach, Franklin, Zatka-Haas \& Diedrichsen, 2014) or to correct for movement errors before they happen (e.g., Desmurget \& Grafton, 2000; Shadmehr, Smith \& 
Krakauer, 2010). During imagery, the same forward models could be used offline, triggered perhaps by sub-threshold motor commands, and allow one to mentally play through how different actions will unfold, without the signals ever reaching the muscles (e.g., Jeannerod, 1994; Jeannerod \& Decety, 1995; Kilteni, Andersson, Houborg \& Ehrsson, 2018).

In these proposals, motor imagery is often described as "neural simulation of action" (e.g., Jeannerod, 2001), “covert execution” (e.g., Scheil, Kleinsorge \& Liefooghe, 2020), and imagined actions are taken as "real actions, except for the fact that they are not executed" (Jeannerod, 2001, p. 103). In essence, these accounts hold that we can imagine our actions because the motoric structures of our brain can, in some form, pretend that the imagined actions are currently executed, and project their perceptual consequences into our imagination, so that we can watch them unfold in front of our mind's eye. Imagination therefore has the same timing, is governed by the same regularities, and activates largely overlapping brain structures as overt action.

\section{A different view on motor imagery}

The previous section describes the "standard" conception of motor imagery. In this article, we would like to advance a markedly different conceptualization, which turns the proposed relationship on its head, and which - as we argue further below - provides a closer match to the extant data. On this view, the links between imagery and overt action do not arise because action imagery is intrinsically motoric, but - conversely - because the mechanisms we use to control our voluntary behaviour are intrinsically imaginistic. In other words, the observed overlaps emerge not because motor imagery recruits motor-based resources but because every action we execute is planned, initiated and controlled through an imagery-like process (e.g., Colton, Bach, Whalley \& Mitchell, 2018; Hommel, 2009; Hommel, Müsseler, 
Aschersleben, \& Prinz, 2001; Janczyk \& Kunde, 2020; Pfister, 2019; Pfister, Janczyk, Wirth, Dignath, \& Kunde, 2014; Prinz, 1997).

This proposal is not new. In fact, it is the classic solution of the ideomotor theorists (e.g., Carpenter, 1852; James, 1890; Harleß, 1861; Lotze, 1852) to the puzzle of how people can achieve voluntary control over their body movements, given that they have very little actual insight into the actual working of their motor apparatus. Even the simplest act of reaching and grasping requires complex coordination of a multitude of muscles, most of which we are not aware of, including those in our back that prevent us from falling forward when we extend our arm. The reader could ask themselves, for example, where in fact the muscles they use to control their finger movements are located, what produces the sounds when they snap their fingers, or how they make a bicycle go left or right. Surprisingly, most people answer these questions incorrectly, even though they have performed these actions many times (see Footnote $^{1}$ for the answers). This "executive ignorance" (Turvey, 1977) into the motor activities that make up our daily lives was neatly summarized by William James: "we are only conversant with the outward results of our volition, and not with the hidden inner machinery of nerves and muscles which are what primarily sets it at work" (1890, p. 499).

Ideomotor theorists argue that imagery is the trick that people use to gain control of a motor apparatus that is essentially a black box to them. The idea is that people do not try to - and actually cannot - directly control their "hidden machinery of nerves of muscles" (James, 1890, p. 499). They can only ever bring to mind a mental image of the "outward results" that they want to achieve, which then activates the motor patterns which will bring this result about. The mechanisms that underlie this transformation of imagery into action are surprisingly simple and based on established laws of associative learning. Accordingly, human agents associate, through a lifetime process of self-observation, the different 
efferent/motor activities that they produce, at first accidentally ('motor babbling'), with the perceptual effects these activities reliably cause (James, 1890; Prinz, 1997; Hommel \& Elsner, 2001). In short, they learn how their body movements look, feel, and sound, and how they affect the environment.

The main argument is that as soon as a behaviour and its likely effects are robustly associated, the mere intention to produce any perceivable effect activates the motor pattern to which it was associated by previous experience. Thus people can purposefully control their black box motor apparatus, merely by thinking of the perceptual effects they wish to achieve: We imagine what it looks like to move our fingers, we bring to mind the sound of our fingers snapping, or where we want our bicycle to go next - and by the previously formed associations the corresponding efferent activities are recollected, without us ever needing to know how they internally realized: imagery of the intended "outward results" is enough to elicit the motor behaviours itself.

While such proposals of effect-based action control have been only rarely connected to the phenomenon of motor imagery, they form the core of modern accounts of effect-based action control (for reviews, see Shin, Proctor, \& Capaldi, 2010, Pfister, 2019), and are captured by recent ideas of action control through internal (inverse and forward) models (Wolpert, 1997) and active inference (Adams, Shipp, \& Friston, 2013). They are supported by a large body of evidence. A full review is not possible here (see Pfister, 2019), but studies have shown that any manipulation that helps people bring to mind the effects of their actions indeed activates the motor behaviours themselves, in line with the idea that both are closely associated. For example, participants execute actions more quickly when primed, just before execution, with an image of the finger (or other body part) movements they should make (e.g., Brass, Bekkering, \& Prinz, 2001; Bach, Peatfield, \& Tipper, 2007), even if this prime is only 
anticipated but not perceived (Kunde, Koch, \& Hoffmann, 2004; see Badets, Koch, \& Philipp, 2016 for review). In some cases, this priming is enough to inadvertently cause participants to execute actions they have been asked to withhold (Colton et al., 2019). Even distal perceptual consequences of one's actions (like a sound elicited as result of a particular button press) prime the motor behaviours that usually bring them about, provided they have been robustly associated before (e.g., Hommel \& Elsner, 2001; Ziessler, Nattkemper \& Vogt, 2012).

Neuroimaging research over the last decades is also consistent with the proposed tight coupling of motor and effect-related representations. In the last decades, it has become clear that, throughout the cortical hierarchy, neuronal populations code for both, efferent (motor) activities and the afferent (perceptual) states they produce, to the extent that it has become increasingly difficult to delineate purely afferent or efferent regions. Regions in early and late visual cortex, for example, previously thought to play purely perceptual roles, have been found to be involved in action planning, and specifically to encode the effects one wants to cause with one's actions (Kühn, Keizer, Rombouts, \& Hommel, 2011; van Steenbergen et al., 2017; Zimmermann et al., 2017; Zimmermann, Verhaegen, de Lange, \& Toni, 2016). A similar "common coding" (Prinz, 1997) of perceptual and motor components also exists in the parietal lobe (e.g., Oosterhof, Tipper \& Downing, 2012; Monaco, Malfatti, Culham, Cattaneo \& Turella, 2020), and the premotor cortex, where neurons have been identified that code actions equally when they are executed and when their effects are perceived, both visually and auditorily (e.g., di Pellegrino, Fadiga, Fogassi, Gallese \& Rizzolatti, 1992; Gallese, Fadiga, Fogassi \& Rizzolatti, 1996; Kohler, et al., 2002). Even the primary motor cortex, often implicated in motor output exclusively, seems to play perceptual roles by representing the anticipated proprioceptive/kinaesthetic consequences of one's behaviour (Naito, 2004; Gandolla et al., 2014; de Lange, Rahnev, Donner \& Lau, 2013), and it has been 
argued that the motor commands it sends to the spinal cord are, in fact, nothing else than proprioceptively coded goal states for one's limbs (e.g., Adams, Shipp, \& Friston, 2013).

Together therefore, there is ample evidence from both experimental psychology and neuroscience that actions are represented in terms of their intended effects, which are then translated into the motor behaviours to which they are associated. If these ideas of effectbased action control are taken seriously, they lead to a subtle but - in our minds illuminating re-conceptualization of motor imagery. Motor imagery, in such frameworks, does not rely on neural re-use of execution-related motor units, but instead reflects specifically the perceptual process through which people plan and initiate their action: the bringing to mind of the goal state - the effects - an actor wants to achieve: how they want their actions to look, sound and feel, when they are carried out.

The difference to overt action is only that this imaginistic process is decoupled from the motor apparatus. Several - not mutually exclusive - alternatives exist how this decoupling may work. One possibility is that efferent activities are somehow inhibited (e.g., Guillot, Di Rienzo, MacIntyre, Moran, \& Collet, 2012; Rieger, Dahm, \& Koch, 2017; Di Rienzo et al., 2014; Berthoz, 1996). Another, perhaps more likely explanation from the perspective of ideomotor thinking, is that the activation threshold to trigger associated motor pattern is deliberately upregulated to prevent an automatic outflow of efferent activity (see also, Berthoz, 1996). In this way, people can plan/imagine actions freely, without being in danger of inadvertently releasing the associated motor behaviours. In fact, this increase of the execution threshold might be the very reason for why agents can become aware of their action imagery at all. During most everyday activities, we move our body without experiencing the vivid images we use to control them. This suggests that even very weak, subliminal action images can drive motor behaviour effectively, and this has indeed been 
supported empirically (Kunde, 2004; Linser \& Goschke, 2007). From an ideomotor perspective, the upregulation of the motor threshold may therefore be precisely what makes motor imagery possible. It allows people to imagine strongly enough to be consciously experienced while still not eliciting overt behaviour.

A recent series of experiments (Colton et al., 2018) tested the idea that imagined actions are nothing else than planned actions that are activated just below the execution threshold. Participants were asked to imagine - but not execute - different sequences of finger movements. In some trials, we unexpectedly strengthened this imagination through a visual cue that showed the effects of the action they currently imagined (e.g., a specific finger depressing), in the hope that this surprising additional activation would drive the action super-threshold and cause its involuntary release. This is exactly what was found. When imagery and visual cues were congruent and could therefore combine, participants found themselves sometimes execute actions they were asked to withhold. If, however, the visual cue did not match what was imagined, the likelihood of such an accidental action slip was if anything reduced (for similar evidence, see Kunde et al., 2004; Maslovat, Chua \& Hodges, 2013).

This conceptualization of motor imagery as sub-threshold effect imagery differs from conventional accounts in that it does not require information from output-related components of the motor apparatus. Instead, it links imagery specifically to the planning-related processes (see also Glover and Baran, 2017; Jeannerod, 1995). We can simply imagine actions because we have done them over and over in our daily life and internalized how they look, sound, and feel. From then on, we can then simply recall this perceptual knowledge to produce a vivid experience of the actions we want to imagine. 
Importantly, while this account does not require a contribution of motoric knowledge, it does not imply that imagery cannot make use of it. In a radical ideomotor view, efferent ("motor") activities are bidirectionally associated to the effects they will cause (cf. Hommel et al., 2001; Hommel and Elsner, 2001; Hecht, Vogt \& Prinz, 2001). Via these links, imagining or intended a specific effect can bring about the associated motor behaviours, but - once they are selected - the activated motor behaviours can also activate the effects they will most likely cause. This is functionally important because the effects used to select a motor behaviour and those it will cause do not need to be identical; any action will bring with itself changes that have not been explicitly intended. For example, one may intend to grasp a coffee mug, including the target location at which the hand should end and how the hand trajectory will look and feel like, but without anticipating the tactile experience of one's pullover's sleeve moving across the forearm, when carrying out that movement.

Through these links, any motor behaviour that is activated may therefore enrich or further constrain imagery with what is most likely to happen when it is executed. This associative activation of the effects an action will also cause might be called a "prediction" and is functionally equivalent to the output of a forward model (cf. Kawato, 1999). It therefore fully implements the main functional mechanism of conventional accounts of imagery, through with (subliminal) motor activation can project ahead which perceptual effects it will most likely cause. The difference is that, in effect-based models, this motor-based prediction is not the default mode. Instead, it considered only as an additional, optional step, that one can draw upon only after motor activities have been accessed through the intended perceptual changes in the first place. There is still no pure "motoric" imagination that is not triggered by a prior imagination of the intended action outcomes. However, once a specific motor behaviour that implements these desired effects is selected, it can be supplemented or adjusted through associations to the effects this motor behaviour will also have, if carried out. 
We believe that such effect-based accounts of motor imagery solve (or sidestep) most problems and inconsistencies in standard accounts. Note for example that, in standard accounts, imagery emerges from motor commands being fed to a forward model-like mechanism, which then plays through the sequence of perceptual consequences these actions will achieve. What is usually left open is where these motor commands come from in the first place. Why, if one already knows the sequence of movements one wants to imagine, is it necessary (a) to identify the precise motor command that brings about this imagination, and (b) how is the relevant chain of motor commands selected, so that it matches this (sometimes quite complex) imagination goal? This ambiguity is even more severe if one considers that the proposed mechanisms (i.e., forward models, efference copies) that serve as the basis for this imagination, are assumed to have evolved to predict the outcomes of actions that the organism does indeed execute (i.e., for anticipatory error control or filtering out expected stimulation). How then is it possible (c) to fool these mechanisms into completing the same job for actions that one does decidedly not execute, because one only wants to only imagine them? Effect-based accounts of motor imagery solve, or sidestep, all these problems by providing a straightforward associative account of how action imagery can affect the motor apparatus, and how, in turn, motoric activity can feed back into what people imagine.

\section{Accounting for the evidence}

We believe that a re-formulation of motor imagery as action planning decoupled from execution has various conceptual advantages over standard accounts. Here, we briefly review how the major findings, which are usually taken as evidence for the standard account, are explained by effect-based accounts of motor imagery. 
Motor imagery elicits muscular activity. One central finding is that motor imagery often engenders measurable activation in the muscles involved in the action (e.g., Jacobson, 1931; 1932; Guillot et al., 2007). The traditional explanation is that this shows the working of an execution-related process, such as the subliminal activation of the motor routines that - via forward modelling - supply imagery with the relevant "material" to play through, such as the precise kinematics or timing of the action one imagines. In effect-based accounts, the explanation of this motor outflow is more straightforward. It simply reflects that the neuronal codes that make up one's imagery of action are the same ones that one would use to initiate via the formed associations - the intended motor behaviours. In such views, it is not surprising that some sub-liminal activation would persist and reach the relevant muscles, even if the motor threshold itself is not passed and no full action is released. In the words of William James "every mental representation of a movement awakens to some degree the actual movement which is its object" (James, 1890). However, these activations may therefore indicate that some form of action imagery takes place, but they do not necessarily play a functional role in the process.

Several findings suggest that this is exactly what is going on. Several studies show striking dissociations between imagery and motor processes. It has long been observed, for example, that muscular responses during mental practice are often only loosely related to the actions one tries to imagine (Heuer, 1985), that motor imagery can elicit motor-like brain activity even if motor output is fully controlled (Zabicki et al., 2017), and that motor imagery typically captures the (ideal) actions one would want to carry out but less so the actual motor behaviour, such as the errors one will make (e.g., Dahm \& Rieger, 2019; Rieger, Martinez \& Wenke, 2011). Such dissociations are problematic for accounts in which motor processes are the causal driver of action imagery, but they are of course fully in line with effect-based views where imagery is primarily perceptually driven, reflecting one's prior knowledge of 
how these behaviours should look, sound and feel, and where motor involvement can enrich this imagery in some tasks but is by no means required (see above).

Other studies show that imagery of non-motor behaviour is often just as effective in causing overt behaviour as that of the motor behaviour itself, if this non-motor behaviour represents possible effects one can cause. One only needs to take a pendulum in one's hand and image it is swinging: one's hand will produce these swings, even though one does not imagine the hand itself moving and is in fact unaware that it does so (Chevreul, 1833; Easton \& Shor, 1975, 1976, 1977). Such findings can be replicated in lab-situations, where visualizing certain shapes is enough to induce a tendency to reproduce these shapes with one's hands, even if these shapes belong to objects that one never has interacted with before (e.g., the circle shape of the moon), making any action imagery unlikely (Bach, Griffiths, Weigelt \& Tipper, 2011). Both findings are much more in line with the idea that motor output during imagery is ultimately driven by bringing to mind the intended action effects, rather than the motor behaviours themselves.

A final example is tool use. For many tools, the effects they produce are clearly dissociable from the motor behaviours that cause them (e.g., opening a hand causes scissors to close and vice versa). Importantly, when people plan such actions, then bringing to mind these effects suffices to elicit the relevant motor behaviours, even if they are opposite to what was imagined (Müsseler, Kunde, Gauspohl, \& Heuer, 2008), and the neuronal coding of these actions seems to occur to a large extent in such an effect-based rather than motoric format (e.g., Ferrari, Rozzi \& Fogassi, 2005). For example, imaging a clockwise rotation of an object facilitates counter-clockwise manual rotations of a steering wheel, provided this produce an intended clockwise rotation of the task-relevant visual object (which applies to current displays in aviation, Janczyk, Pfister, Crognale, \& Kunde, 2012). These findings therefore 
provide clear evidence that motor imagery - like action planning - occurs not in terms of motoric outflow, but in terms of the effects one wants to achieve with one's actions (Hommel et al. 2001; Prinz, 1997).

Action execution can help or hinder imagery. A second class of important findings is that executing motor behaviours disrupts imagery of different actions, but helps imagery of similar ones (e.g., Wohlschläger, 1996; 2001; Callow et al., 2006; for a review, see Guillot, under review). In the standard view, these effects emerge because carrying out an action occupies one's execution-related resources so that they cannot be deployed for motor imagery, or because a forward model that is fed by mismatching motor commands will also conjure up mismatching mental images. In principle, these explanations can also work in frameworks of effect-based action control, as they assume not only that effect imagery triggers motor behaviour, but also that motor behaviour, in turn, is associated to the relevant perceptual effects it will bring about (e.g., Hommel et al., 2001; Müsseler \& Hommel, 1997). Executing movements that differ from imagination will therefore by necessity also conjure up inappropriate effect images, therefore interfering with the imagery process.

However, we suspect that there might be a simpler explanation. Executing a movement is not perception-free. Even if people are not looking at body parts that they move, they still receive (proprioceptive, tactile, etc.) feedback from their limbs and muscles. And of course, this feedback would also not be congruent with the perceptual effects one imagines, so that interference can arise not only predicted feedback that mismatches imagery, but also from the actual feedback that is inconsistent with the current imagery task. A classic example is that people can mentally rotate an object more easily if they rotate their hands in the same direction, compared to the opposite direction (Wohlschläger, 1996; 2001; for a review of similar findings, see Guillot, under review). This does not necessarily reflect the anticipation 
of incongruent movement effects, but also the multisensory associations of clockwise proprioceptive feedback with clockwise visual motions (cf. de Lange, Helmich \& Toni, 2006, Shenton, Schwoebel \& Coslett, 2004).

Findings from deafferented patients - who receive no proprioceptive feedback - provide direct evidence for such multisensory associations. Usually, people find it difficult to draw when they only see the mirror image of their drawing hand, as the mismatch between visual and proprioceptive information makes leftward movements of one's body look rightwards in the mirror and vice versa. To compensate, neurotypical agents often try to ignore such mismatching proprioceptive feedback, thereby inducing 'functional haptic neglect' (Liesner \& Kunde, 2020; Heuer \& Rapp, 2012). Strikingly, this performance drop is absent in deafferented patients, showing that the interference indeed emerges, at least in part, on a perceptual (proprioceptive to visual) locus (Lajoie et al., 1992).

Temporal and kinematic similarities between imagery and action. A central piece of evidence for motoric accounts is the tight temporal link between overt and imagined action (e.g., Decety, Jeannerod, Prablanc, 1989; Wohlschläger \& Wohlschläger, 1998) and the finding that both are governed by similar regularities, such as Fitts law, or the two-thirds Power law between speed and curvature of biological movements (Decety \& Jeannerod, 1995; Karklinsky \& Flash, 2015; Papaxanthis et al, 2012). As these constraints are argued to emerge from the motor system itself, their re-occurrence in imagery is taken as evidence that imagery must reflect a "read out" of a motoric process.

While such perceptual-to-motor effects can in principle be accounted within effect-based accounts (see above), we do not believe that these findings can be taken at face value. Typical research participants have ample perceptual experience with their own behaviour (and that of others) throughout their lifetime, and it would be no surprise that their imagination - and 
ability to plan their actions - has internalized these regularities and takes them into account. A strong case for motoric readout could therefore only be made in cases where imagery can be shown to be governed by a regularity that (a) reflects constraints from the motor apparatus, and which (b) participants had no prior perceptual experience with. We are not aware of any such demonstration. Instead, several studies have suggested that regularities originally attributed to the motor apparatus may in fact reflect perceptual or imaginistic constraints. For example, it is well-established that people find it almost impossible to perform particular irregular bimanual movements, for example when having to describe three circles with one hand while the other performs four (Kelso, 1995). These limitations have been put down to constraints of the motor apparatus, such as a synchronization of motor commands in the spinal cord. However, in an impressive series of demonstrations, Mechsner and colleagues (Mechsner, Kerzel, Knoblich \& Prinz, 2001; Mechsner, 2003) have shown that these phenomena may instead reflect constraints of imaginistic action planning. The limitations were alleviated when participants were given help to perceptually coordinate - imagine these actions, so that "impossible" movements suddenly became possible.

In addition, many of the initial findings of tight temporal overlaps between overt and imagined actions have been recently challenged, with several showing either consistent overor under-estimation of the timings of imagined relative to overt actions, depending on task and stimulus contexts (e.g., Reed, 2002; for a review, see Guillot \& Collet, 2005). This temporal elasticity is problematic for approaches that assume that people's mental simulation of actions emerges from a subliminal play-through of the underlying motor routines, which should then closely match their actual time course. However, if imagery reflects a planning process that is decoupled from execution resources (see also Jeannerod, 1995; Glover \& Baran, 2017), such differences are not surprising, and indeed expected, if one assumes that people flexibly decide to either faithfully follow their memories of action execution, going 
through their major functional stages only, or speed up or slow down specific steps to extract the information required for their particular imagery task or goal.

Imagery and action activate similar brain regions. A final important piece of evidence for motoric accounts is the tight overlap between brain structures activated by overt and imagined action (for reviews, see Lotze \& Halsband, 2006; O’Shea \& Moran, 2017). In our view, an interesting, but often ignored, aspect of these observations is that this overlap is primarily observed in structures associated with action planning - like premotor and parietal cortices - but it is much less robust in execution related structures, like the primary motor cortices, basal ganglia and cerebellum (for review and meta-analysis, see O'Shea \& Moran, 2017; Hétu et al., 2013; Munzert, Lorey \& Zentgraf, 2009). For motoric accounts of action imagery, such a finding should be puzzling, but it is of course exactly what would be predicted by accounts of effect-based action control, where action imagery is conceptualized precisely as a shift towards planning away from execution-related resources (see also Jeannerod, 1995; Baran \& Glover, 2017).

An elegant study confirmed this link between motor imagery and planning-related instead of execution-related processes, while controlling for actual body movement (Raffin et al., 2012). The authors asked amputees, who still subjectively felt the presence of their phantom limbs, to either really move or imagine moving their absent limbs. No movement - and no proprioceptive feedback - was of course present in either case. Nevertheless, there were clear differences in brain activation. The execution instruction more strongly activated primary somatosensory and motor cortices and the anterior lobe of the cerebellum. In contrast, during imagination there was more activity in the parietal and occipital lobes, and the posterior cerebellum (see also Hanakawa, Dimyan \& Hallett, 2008). These regions matched those when the patients really moved, or imagined moving, their still intact limbs. This shows both: 
that motor execution and imagery are indeed different, and that these differences map onto the proposed shift from motor to planning-related resources of effect-based control.

\section{Open questions and outlook}

Motoric views of motor imagery have left several puzzles open that have either been unresolved, or not been addressed. An effect-based view provides a new view on them, and in many cases - clear avenues how they can be resolved, including testable predictions. Below we provide a first sketch for some of these open questions.

When does imagery draw upon motor resources? In effect-based accounts, motor imagery primarily reflects the planning stages of actions, which occur without recourse to the motor apparatus, based simply on one's perceptual experience of one's own actions. There is no need for imagery to draw upon knowledge encoded by the motor apparatus, for example, by accessing a forward model that is fed with the motor commands of actions one could execute. As pointed out above, this does not mean that such an influence is not possible, however. The simple associative architecture of effect-based accounts predicts not only that the effects we intend will elicit the motor behaviours that will bring them about, but also that the motor behaviours, once selected, activate the effects they will produce (e.g., Hommel et al., 2001; Müsseler \& Hommel, 1997). These latter pathways therefore allow one to derive how one's behaviours will play out, providing a secondary "motoric" path to imagery.

An important question is under what circumstances this secondary route is made use of. We suspect that this happens specifically when the effects usually used to plan a particular action do not correspond to the information required by the imagery task. For example, it is typically assumed that the effects agents use to control their behaviour are coded in a 
relatively sparse manner, capturing mainly the intended "distal" bodily or environmental effects (Hommel et al., 2001), the required transitions between current and intended perceptual states (Kunde, Schmidts, Wirth, \& Herbort, 2017), or the functional sub-steps of an action sequence (Schack \& Mechsner, 2006). As the specific timing and kinematic of one's skilled movements emerge, to a large extent, from biomechanical constraints within the muscular and skeletal system during execution, they are most likely not represented on this planning level, unless the skill specifically requires controlling them (e.g., in dance, or for specific unusual actions cf., Mechsner et al., 2001). If this is correct, then the motoric route might be necessary whenever the task requires access to such action features that are not captured by the perceptual goal states people use to control their actions.

Accordingly, for motor behaviour where timing and kinematics do not need accurate endogenous controlled, imagery should similarly only engender limited activation in brain structures involved in action execution, and outflow to muscles should be limited. However, as soon as one's imagery task requires deriving accurate timing, precise kinematics, or access to internal bodily feedback, such motoric effects should emerge also for these movements. Several studies show that motoric indices of motor imagery indeed emerge for such instructions (e.g., Stinear et al., 2006), and this notion is also well supported by the implicit practice of motor imagery researchers. To ensure that motoric activations are found, researchers often instruct their participants to focus on the proprioception and kinesthesis, or the sensations of the actions, not just their visual representation, or tasks are made sufficiently complex to require such forward modelling.

Why is mental practice effective? One rarely addressed conflict in standard approaches is the evidence that mental practice is particularly effective in conditions in which it should not be. If mental practice relies on a subliminal play-through of motor routines, why is it more 
effective in sports with strong cognitive instead of motor components (like chess, golf, etc; see Ryan \& Simons, 1981; 1983 for the original distinction), why are effect sizes usually smaller for purely motoric ones (for meta-analyses, see Driskell et al., 1994; Toth et al., 2020; Weinberg, 2008), and why is the myoelectric activity that accompanies it only loosely connected to the practiced movements (Heuer, 1985)? The findings are similar for studies that test how learners are best instructed in a motor skill. It is usually better to instruct people in terms of the consequences - the effects - they want to achieve their actions (e.g., focus on hitting the darts board in the bullseye) than reminding them of the proper motoric technique (pulling back the hand, flick of the finger, etc), which can even be counter-productive (for review, see Wulf, 2013).

If one assumes that mental practice is effective because it affords a subliminal playthrough of motor routines, stronger benefits in the absence of motoric instructions should be at least slightly puzzling. However, such results are of course precisely what would be expected under and effects-based imagery view. In such views, playing through one's action would simply help in deriving better plans for one's actions, without necessary input from efferent/motor activities. Repeated effect imagery alone can (a) make these effect images more accessible so that they are more easily brought to mind in the performance situation, it (b) can make these images sharper and more salient so that they become better drivers of muscular activities, and, especially for longer action sequences, it might (c) help one to imagine different sub-steps and schedule them more efficiently.

Consistent with these views, recent work shows that athletes represent skilled actions (such as a golf putt) in a hierarchical manner (Schack \& Mechsner, 2006). These perceptual-cognitive representations describe the interrelations between the action's functional parts, with each of them being linked to particular perceptual effects, sharing more or less effects with other 
functional sub-actions, and thus resulting in functional groupings for action control (Schack, 2004, 2020; Bläsing, Tenenbaum, \& Schack, 2009). When novices physically practice a motor action physically - such as a golf putt - these mental representations become stabilized and structurally revised into functional groups (e.g., a preparation phase, a clubhead-ball impact phase, and an attenuation phase after the putt; Frank, Land, \& Schack, 2013; Frank, 2016). From an effect-based view, mental practice can both stabilize these acquired skill structures or cause similar revisions as physical practice (Frank, 2014; Schack, 2006; Schack \& Frank, 2019). Indeed, when novices practice mentally, their perceptual-cognitive representation system shows similar structural changes (Frank, Land, Popp, \& Schack, 2014). Strikingly, while changes in novices' representations are reflected in overt behavioural changes after physical practice, they do not necessarily transfer into better performance after mental practice, unless athletes had some prior physical experience in which the different parts of a skill's structure were associated with the relevant motor activities that reliably produce these effects (Frank et al., 2014). In this way, the effect-based approach suggests some clear boundary conditions when mental practice should be effective.

An interesting avenue for future research are attempts to enrich actions by additional perceptual feedback (e.g., sonification for the case of auditory feedback, e.g., Effenberg, Fehse, Schmitz, Krueger, \& Mechling, 2016). The idea here is to provide agents with auditory feedback of their body movements that is more discriminable than the perceptual (visual, interoceptive) feedback these movements naturally produce. This should not only enable agents to distinguish motor patterns that were not discriminable without such feedback, but also provide them with another means to imagine their movements. They can imagine how it sounds to move in specific ways, rather than just visually or proprioceptively imagine doing so, just as musicians plan their playing in terms of these musical effects instead of body movements (Brooks, 1995; Drost, Rieger, Brass, Gunter, \& Prinz, 2005ab). 
Integration of motor with non-motor imagery? Most everyday tasks require the integration of own action capabilities with the characteristics of the environment. For example, for a successful golf swing, the golfer needs to coordinate their (upper) body and limbs with respect to the grass surface, the club one uses, direction and speed of the wind, among other factors. Similarly, during everyday tool use, people need to consider motoric features (e.g., the flexion and extension of arms during hammering), relative to the mechanical properties of the tool and the object it is applied to (e.g., a nail vs. a pane of glass) (see Osiurak \& Badets, 2016, for a review of mechanical problem solving in tool use).

An account that can describe how such actions are planned, or imagined, needs a means of integrating motoric with extra-motoric information form the physical and social environment (see Bach et al., 2005; 2010; 2014 for similar ideas in an action observation context). It is difficult to see how such integration would be implemented in purely motoric stimulation views, as it is unclear how non-motoric knowledge about the physical behaviour of objects could interact with motoric knowledge of one's own action (but see Schubotz, 2007 for an interesting approach). However, if one assumes, as effect-based accounts do, that action planning generally relies on the same cognitive codes as imagery of physical events, such an integration is no mystery. One's perceptual learning about the behaviour of physical system and about one's own motor behaviour can then be seamlessly integrated.

Some recent evidence from monkey single cell recordings studies shows that the perceptual systems indeed support such integration. In one study, monkeys first had to identify a relevant object, then trace its outline, and then direct an action towards its endpoint. Early visual cortex played a central role in each of these steps, initially highlighting the relevant object, then tracing its outline, and then its endpoint, which then served as the basis for eye movements towards it (Moro, Tolboom, Khayat, \& Roelfsema, 2010, Roelfsema, Khayat, \& 
Spekreijse, 2003; for a review, see Roelfsema \& de Lange, 2016). These findings are therefore exactly in line with effect-based account, where relevant paths are first perceptually organized, and the exact same representations can then form as basis for action control. We are looking forward to future studies that hopefully show such interactions in human participants.

\section{Conclusions}

We have briefly sketched how motor imagery can be conceptualized in effect-based views of action control. These views hold that actions are planned in term of their intended perceptual effects - how a successful action should look, feel, and sound -, which are then made reality by automatic motor processes. In such views, motor imagery reflects this imaginistic planning process, which is decoupled from execution. Imagery experience simply reflect the recall of one's perceptual (visual, auditory, proprioceptive) experience with one's actions, without requiring efferent (motoric) contributions. If these views are taken seriously, the term "motor imagery" becomes a misnomer; "effect imagery" might be a better description of how people imagine - and plan - their (motor) actions. The evidence reviewed here makes an explicit case for such an account.

\section{References}

Adams, R. A., Shipp, S., \& Friston, K. J. (2013). Predictions not commands: active inference in the motor system. Brain Structure and Function, 218(3), 611-643. 
Anderson, M. (2010). Neural reuse: A fundamental organizational principle of the brain. Behavioral and brain sciences, 33(4), 245.

Bach, P., Allami Khalaf, B., Tucker, M., \& Ellis, R. (2014). Planning-related motor processes underlie mental practice and imitation learning. Journal of Experimental Psychology: General, 143(3), 1277-1294.

Bach, P., Griffiths, D., Weigelt, M., Tipper, S.P. (2010). Gesturing meaning. Non-action words activate the motor system. Frontiers in Human Neuroscience, 4:214.

Bach, P., Knoblich, G., Gunter, T.C., Friederici, A.D., Prinz, W. (2005). Action Comprehension: Deriving Spatial and Functional Relations. Journal of Experimental Psychology: Human Perception \& Performance, 31(3), 465-479.

Bach, P., Nicholson, T., \& Hudson, M. (2014). Bach, P., Nicholson, T., \& Hudson, M. (2014). The affordance-matching hypothesis: how objects guide action understanding and prediction. Frontiers in Human Neuroscience, 8.

Bach, P., Peatfield, N.A., \& Tipper, S.P. (2007). Focusing on body sites: the role of spatial attention in action perception. Experimental Brain Research, 178, 509-517.

Bach, P., Peelen, V.M., Tipper, S.P. (2010). On the role of object information in action observation: an fMRI study. Cerebral Cortex, 20(12), 2798-2809.

Berthoz, A. (1996). The role of inhibition in the hierarchical gating of executed and imagined movements. Cognitive Brain Research, 3(2), 101-113.

Bläsing, B., Tenenbaum, G., \& Schack, T. (2009). The cognitive structure of movements in classical dance. Psychology of Sport and Exercise, 10, 350-360. 
Brooks, R. W. (1995). Mental practice and the musician: A practical approach to practice. Update: Applications of Research in Music Education, 13(2), 4-8.

Callow, N., Roberts, R., \& Fawkes, J. Z. (2006). Effects of dynamic and static imagery on vividness of imagery skiing performance, and confidence. Journal of Imagery Research in Sport and Physical Activity, 1, 1-15.

Carpenter, W.B. (1852). On the Influence of Suggestion in Modifying and directing Muscular Movement, independently of Volition. Royal Institution of Great Britain, (Proceedings), 147-153.

Chevreul, M. E. (1833). Lettre à M. Ampère sur une classe particulière de mouvements musculaires. Review Des Deux Mondes, (2), 258-266.

Colton, J. Bach, P. Whalley, B., \& Mitchell, C. (2018). Intention insertion: activating an action's perceptual consequences is sufficient to induce non-willed motor behaviour. Journal of Experimental Psychology: General, 147(8), 1256-1263.

Dahm, S. F., \& Rieger, M. (2019). Is imagery better than reality? Performance in imagined dart throwing. Human movement science, 66, 38-52.

Dahm, S. F., \& Rieger, M. (2019). Is imagery better than reality? Performance in imagined dart throwing. Human movement science, 66, 38-52.

de Lange, F. P., Helmich, R. C., \& Toni, I. (2006). Posture influences motor imagery: an fMRI study. Neuroimage, 33(2), 609-617.

de Lange, F. P., Rahnev, D. A., Donner, T. H., \& Lau, H. (2013). Prestimulus oscillatory activity over motor cortex reflects perceptual expectations. Journal of Neuroscience, 33(4), 1400-1410. 
Decety, J., \& Jeannerod, M. (1995). Mentally simulated movements in virtual reality: does Fitt's law hold in motor imagery?. Behavioural brain research, 72(1-2), 127-134.

Desmurget, M., \& Grafton, S. (2000). Forward modeling allows feedback control for fast reaching movements. Trends in cognitive sciences, 4(11), 423-431.

Di Rienzo, F., Guillot, A., Daligault, S., Delpuech, C., Rode, G., \& Collet, C. (2014). Motor inhibition during motor imagery: a MEG study with a quadriplegic patient. Neurocase, 20(5), 524-539.

Driskell, J., Copper, C., \& Moran, A. (1994). Does mental practice enhance performance? Journal of Applied Psychology, 79, 481-492.

Drost, U. C. Rieger, M. Brass, M. Gunter, T. C. Prinz, W. (2005a). Action-effect coupling in pianists. Psychological Research 69, 233-241.

Drost, U. C. Rieger, M. Brass, M. Gunter, T. C. Prinz, W. (2005b). When hearing turns into playing: Movement induction by auditory stimuli in pianists. Quarterly Journal of Experimental Psychology 58A, 1376-1389.

Easton, R. D., \& Shor, R. E. (1975). Information processing analysis of the Chevreul pendulum illusion. Journal of Experimental Psychology: Human Perception and Performance, 1(3), 231-236. https://doi.org/10.1037/0096-1523.1.3.231

Easton, R. D., \& Shor, R. E. (1976). An Experimental Analysis of the Chevreul Pendulum Illusion. The Journal of General Psychology, 95(1), 111-125. https://doi.org/10.1080/00221309.1976.9710871

Easton, R. D., \& Shor, R. E. (1977). Augmented and Delayed Feedback in the Chevreul Pendulum Illusion. The Journal of General Psychology, 97(2), 167-177. 
https://doi.org/10.1080/00221309.1977.9920835

Effenberg, A. O., Fehse, U., Schmitz, G., Krueger, B., \& Mechling, H. (2016). Movement Sonification: Effects on Motor Learning beyond Rhythmic Adjustments. Frontiers in Neuroscience, 10(149), 67.

Ferrari, P. F., Rozzi, S., \& Fogassi, L. (2005). Mirror neurons responding to observation of actions made with tools in monkey ventral premotor cortex. Journal of cognitive neuroscience, 17(2), 212-226.

Frank, C. (2014). Mental representation and learning in complex action: A perceptualcognitive view on mental and physical practice ( $\mathrm{PhD}$ thesis, Dr. rer. nat.). Universität Bielefeld.

Frank, C. (2016). Learning a motor action from within: Insights into the development of one's action representation with mental and physical practice. In M. Raab, P. Wylleman, R. Seiler, A.-M. Elbe, \& A. Hatzigeorgiadis (Eds.), Sport and exercise psychology research from theory to practice (pp. 91-121). Amsterdam: Elsevier.

Frank, C., Land, W. M., \& Schack, T. (2013). Mental representation and learning: The influence of practice on the development of mental representation structure in complex action. Psychology of Sport and Exercise, 14, 353-361.

Frank, C., Land, W. M., Popp, C., \& Schack, T. (2014). Mental representation and mental practice: Experimental investigation on the functional links between motor memory and motor imagery. PLoS ONE, 9, e95175.

Gandolla, M., et al. (2014). Re-thinking the role of motor cortex: context-sensitive motor outputs?. Neuroimage, 91, 366-374. 
Glover, S., \& Baran, M. (2017). The motor-cognitive model of motor imagery: Evidence from timing errors in simulated reaching and grasping. Journal of Experimental Psychology: Human Perception and Performance, 43(7), 1359.

Guillot, A., Di Rienzo, F., MacIntyre, T., Moran, A., \& Collet, C. (2012). Imagining is not doing but involves specific motor commands: A review of experimental data related to motor inhibition. Frontiers in Human Neuroscience, 6, Article 247.

Guillot, A., Lebon, F., \& Collet, C. (2010). Electromyographic activity during motor imagery. In A. Guillot \& C. Collet (Eds.), The neurophysiological foundations of mental and motor imagery (pp. 83-94). Oxford: Oxford University Press.

Guillot, A., Lebon, F., Rouffet, D., Champely, S., Doyon, J., \& Collet, C. (2007). Muscular responses during motor imagery as a function of muscle contraction types. International Journal of Psychophysiology, 66(1), 18-27.

Guillot, A., Moschberger, K., \& Collet, C. (2013). Coupling movement with imagery as a new perspective for motor imagery practice. Behavioral and Brain Functions, 9, 8.

Hanakawa, T., Dimyan, M. A., \& Hallett, M. (2008). Motor planning, imagery, and execution in the distributed motor network: a time-course study with functional MRI. Cerebral cortex, 18(12), 2775-2788.

Harleß, E. (1861). Der Apparat des Willens. Zeitschrift für Philosophie und philosophische Kritik, 38(2), 50-73.

Hecht, H., Vogt, S., \& Prinz, W. (2001). Motor learning enhances perceptual judgment: A case for action-perception transfer. Psychological research, 65(1), 3-14.). Motor 
learning enhances perceptual judgment: A case for action-perception transfer. Psychological research, 65(1), 3-14.

Herwig, A., Beisert, M., \& Prinz, W. (2013). Action science emerging: Introduction and leitmotifs. In W. Prinz, M. Beisert, \& A. Herwig (Eds.), Action science: Foundations of an emerging discipline (pp. 1-33). Cambridge, MA: MIT Press.

Hétu, S., Grégoire, M., Saimpont, A., Coll, M. P., Eugène, F., Michon, P. E., \& Jackson, P. L. (2013). The neural network of motor imagery: an ALE meta-analysis. Neuroscience \& Biobehavioral Reviews, 37(5), 930-949.

Heuer H, Rapp K (2012). Adaptation to novel visuo-motor transformations: further evidence of functional haptic neglect. Experimental Brain Research. 218(1). 129-140.

Heuer, H. (1985). Wie wirkt mentale Übung?. Psychologische Rundschau, 36(4), 191-200.

Jacobson, E., 1931. Electrical measurements of neuromuscular states during mental activities. Am. J. Physiol. 96, 115-121.

Jacobson, E., 1932. Electrophysiology of mental activities. Am. J. Psychol. 44,677-694.

Janczyk, M. \& Kunde, W. (2020). Dual tasking from a goal perspective. Psychological Review, 127(6), 1079-1096.

Janczyk, M., Pfister, R., Crognale, M. A., \& Kunde, W. (2012). Effective rotations: action effects determine the interplay of mental and manual rotations. Journal of Experimental psychology. General, 141(3), 489-501.

Jeannerod, M. \& Decety, J. Mental motor imagery: a window into the representational stages of action. Curr. Opin. Neurobiol. 5, 727-732 (1995). 
Jeannerod, M. (2001). Neural simulation of action: a unifying mechanism for motor cognition. Neuroimage, 14(1), S103-S109.

Jeannerod, M. (2001). Neural simulation of action: a unifying mechanism for motor cognition. Neuroimage, 14(1), S103-S109.

Jeannerod, M. The representing brain: neural correlates of motor intention and imagery. Behav. Brain Sci. 17, 187 (1994).

Karklinsky, M., \& Flash, T. (2015). Timing of continuous motor imagery: the two-thirds power law originates in trajectory planning. Journal of neurophysiology, 113(7), 24902499.

Kawato, M. (1999). Internal models for motor control and trajectory planning. Current Opinion in Neurobiology, 9(6), 718-727.

Kelso, J. A. S. Dynamic patterns: The Self-organization of Brain and Behavior (MIT Press, Cambridge, Massachusetts, 1995).

Kilteni, K., Andersson, B. J., Houborg, C., \& Ehrsson, H. H. (2018). Motor imagery involves predicting the sensory consequences of the imagined movement. Nature communications, 9(1), 1-9.

Kohler, E., Keysers, C., Umilta, M. A., Fogassi, L., Gallese, V., \& Rizzolatti, G. (2002). Hearing sounds, understanding actions: action representation in mirror neurons. Science, 297(5582), 846-848.

Krüger, B., Hettwer, M., Zabicki, A., de Haas, B., Munzert, J., \& Zentgraf, K. (2020). Practice modality of motor sequences impacts the neural signature of motor imagery. Scientific reports, 10(1), 1-13. 
Kunde, W. (2004). Response priming by supraliminal and subliminal action effects. Psychological Research, 68(2), 91-96.

Kunde, W., Schmidts, C., Wirth, R., \& Herbort, O. (2017). Action effects are coded as transitions from current to future stimulation: Evidence from compatibility effects in tracking. Journal of Experimental Psychology: Human Perception and Performance, 43(3), 477.

Lajoie Y, Paillard J, Teasdale N, Bard C, Fleury M, Forget R, et al (1992). Mirror drawing in a deafferented patient and normal subjects: visuoproprioceptive conflict. Neurology. 42: 1104-1106.

Land, W. M., Frank, C., \& Schack, T. (2014). The impact of attentional focus on the development of skill representation in a complex action. Psychology of Sport and Exercise, 15, 30-38.

Liesner M., Kunde W. (2020) Suppression of mutually incompatible proprioceptive and visual action effects in tool use. PLoS ONE 15(11): e0242327.

Linser, K., \& Goschke, T. (2007). Unconscious modulation of the conscious experience of voluntary control. Cognition, 104(3), 459-475.

Lotze H.R. (1852). Medicinische Psychologie oder Physiologie der Seele [Medical psychology or the physiology of the mind]. Leipzig: Weidmann'sche Buchhandlung.

Lotze, M. \& Halsband, U. Motor imagery. J. Physiol. Paris 99, 386-395 (2006).

Lutz, R. S. (2003). Covert muscle excitation is outflow from the central generation of motor imagery. Behavioral Brain Research, 140, 149-163. 
Maslovat, D., Chua, R., \& Hodges, N. J. (2013). When unintended movements "leak" out: A startling acoustic stimulus can elicit a prepared response during motor imagery and action observation. Neuropsychologia, 51(5), 838-844.

Mayer, J., \& Hermann, H.-D. (2019). Kognitives Training im Sport. In J. Schüler, M.

Wegner, H. Plessner (Hrsg.), Sportpsychologie (S. 463-478). Heidelberg: Springer.

Mechsner, F. (2003). Gestalt factors in human movement cordination. Gestalt Theory.

Mechsner, F., Kerzel, D., Knoblich, G., \& Prinz, W. (2001). Perceptual Basis of Bimanual Coordination. Nature, 414, 69-73.

Miall, R. C., \& Wolpert, D. M. (1996). Forward models for physiological motor control. Neural networks, 9(8), 1265-1279.

Miall, R. C., \& Wolpert, D. M. (1996). Forward models for physiological motor control. Neural networks, 9(8), 1265-1279.

Monaco, S., Malfatti, G., Culham, J. C., Cattaneo, L., \& Turella, L. (2020). Decoding motor imagery and action planning in the early visual cortex: overlapping but distinct neural mechanisms. Neuroimage, 218, 116981.

Munzert, J., Lorey, B., \& Zentgraf, K. (2009). Cognitive motor processes: the role of motor imagery in the study of motor representations. Brain research reviews, 60(2), 306-326.

Müsseler, J., \& Hommel, B. (1997). Blindness to response-compatible stimuli. Journal of Experimental Psychology: Human Perception and Performance, 23(3), 861.

Müsseler, J., Kunde, W., Gausepohl, D., \& Heuer, H. (2008). Does a tool eliminate spatial compatibility effects?. European Journal of Cognitive Psychology, 20(2), 211-231. 
O’Shea, H., \& Moran, A. (2017). Does motor simulation theory explain the cognitive mechanisms underlying motor imagery? A critical review. Frontiers in Human Neuroscience, 11,72 .

Oosterhof, N. N., Tipper, S. P., \& Downing, P. E. (2012). Visuo-motor imagery of specific manual actions: a multi-variate pattern analysis fMRI study. Neuroimage, 63(1), 262271.ooste

Papaxanthis, C., Paizis, C., White, O., Pozzo, T., \& Stucchi, N. (2012). The relation between geometry and time in mental actions. PloS one, 7(11), e51191.

Paravlik, A., Slimani, M., Tod, D., Marusic, U., Milanovic, Z., \& Pisot, R. (2018). Effects and dose-response relationships of motor imagery practice on strength development in healthy adult populations: A systematic review and meta-analysis. Sports Medicine, 48, 1165-1187.

Pfister, R. (2019). Effect-based action control with body-related effects: Implications for empirical approaches to ideomotor action control. Psychological Review, 126(1), 153.

Pfister, R., Janczyk, M., Wirth, R., Dignath, D., \& Kunde, W. (2014). Thinking with portals: Revisiting kinematic cues to intention. Cogniton, 133(2), 464-473.

Raffin, E., Mattout, J., Reilly, K. T., \& Giraux, P. (2012). Disentangling motor execution from motor imagery with the phantom limb. Brain, 135(2), 582-595.

Reichenbach, A., Franklin, D. W., Zatka-Haas, P., \& Diedrichsen, J. (2014). A dedicated binding mechanism for the visual control of movement. Current Biology, 24(7), 780785. 
Reiser, M., Büsch, D., \& Munzert, J. (2011). Strength gains by imagination of muscle actions with different ratios of physical to mental training. Frontiers in Psychology, 2, Article 194.

Rieger, M., Martinez, F., \& Wenke, D. (2011). Imagery of errors in typing. Cognition, 121(2), 163-175.

Rieger, M., Martinez, F., \& Wenke, D. (2011). Imagery of errors in typing. Cognition, 121(2), 163-175.

Roelfsema, P. R., \& de Lange, F. P. (2016). Early visual cortex as a multiscale cognitive blackboard. Annual review of vision science, 2, 131-151.

Ryan, E. D., \& Simons, J. (1981). Cognitive demand, imagery, and frequency of mental rehearsal as factors influencing acquisition of motor skills. Journal of Sport Psychology, 3, 35-45.

Ryan, E. D., \& Simons, J. (1983). What is learned in mental practice of motor skills: A test of the cognitive-motor hypothesis. Journal of Sport Psychology, 5, 419-426.

Schack, T. (2004). The cognitive architecture of complex movement. International Journal of Sport and Exercise Psychology, 2, 403-438.

Schack, T. (2006). Mentales Training. In M. Tietjens \& B. Strauss (Hrsg.), Handbuch Sportpsychologie (S. 254-261). Schorndorf: Hofmann.

Schack, T. (2020). Mental representation in action. A cognitive architecture approach. In G. Tenenbaum \& R. Eklund (Eds.), Handbook of Sport Psychology (pp. 513-534). Hoboken: Wiley. 
Schack, T., \& Frank, C. (2019). Ideomotor training. In D. Hackfort, R. Schinke, \& B. Strauss (Eds.), Dictionary of Sport Psychology: Sport, Exercise, and Performing Arts (pp. 138139).

Schack, T., Essig, K., Frank, C., \& Koester, D. (2014). Mental representation and motor imagery training. Frontiers in Human Neuroscience, 8, 328.

Schack, T., Essig, K., Frank, C., \& Koester, D. (2014). Mental representation and motor imagery training. Frontiers in human neuroscience, 8, 328

Scheil, J., Kleinsorge, T., \& Liefooghe, B. (2020). Motor imagery entails task-set inhibition. Psychological research, 84(6), 1729-1738.

Shadmehr, R., Smith, M. A., \& Krakauer, J. W. (2010). Error correction, sensory prediction, and adaptation in motor control. Annual review of neuroscience, 33, 89-108.

Shadmehr, R., Smith, M. A., \& Krakauer, J. W. (2010). Error correction, sensory prediction, and adaptation in motor control. Annual review of neuroscience, 33, 89-108.

Shaw, W.A., 1938. The distribution of muscular action potentials during imaging. Psychol. Rec. 2, 195-216.

Shenton, J. T., Schwoebel, J., \& Coslett, H. B. (2004). Mental motor imagery and the body schema: evidence for proprioceptive dominance. Neuroscience letters, 370(1), 19-24.

Shin, Y. K., Proctor, R. W., \& Capaldi, E. J. (2010). A review of contemporary ideomotor theory. Psychological Bulletin, 136(6), 943.

Simonsmeier, B. A., Frank, C., Gubelmann, H., \& Schneider, M. (2018). The effects of motor imagery training on performance and mental representation of 7- to 15-year old 
gymnasts of different levels of expertise. Sport, Exercise, and Performance Psychology, 7, 155-168.

Smith, D., Collins, D., \& Holmes, P. (2003). Impact and mechanism of mental practice effects on strength. International Journal of Sport and Exercise Psychology, 1(3), 293306.

Stinear, C. M., Byblow, W. D., Steyvers, M., Levin, O., \& Swinnen, S. P. (2006). Kinesthetic, but not visual, motor imagery modulates corticomotor excitability. Experimental brain research, 168(1-2), 157-164.

Toth, A. J., McNeill, E., Hayes, K., Moran, A. P., \& Campbell, M. (2020). Does mental practice still enhance performance? A 24 Year follow-up and meta-analytic replication and extension. Psychology of Sport and Exercise, 48, 101672.

Toth, A., McNeill, E., Hayes, K., Moran, A., \& Campbell, M. (2020). Does mental practice still enhance performance? A 24 year follow-up and meta-analytic replication and extension. Psychology of Sport and Exercise, 48, 101672.

Turvey, M. T. (1977). "Preliminaries to a theory of action with reference to vision," in Perceiving, Acting and Knowing: Toward an Ecological Psychology, eds R. Shaw and J. Bransford (Hillsdale, NJ: Erlbaum), 211-265.

Weinberg, R. (2008). Does imagery work? Effects on performance and mental skills. Journal of Imagery Research in Sport and Physical Activity, 3(1), 1-21. https://doi.org/ 10.2202/1932-0191.1025. 
Wohlschläger, A. (1996). Mental rotation — a case of embodied action. In Embodied Cognition \& Action. Papers from the 1996 Fall Symposium. Technical Report FS-9605 (pp. 139-144).

Wohlschläger, A., \& Wohlschläger, A. (1998). Mental and manual rotation. Journal of Experimental Psychology: Human perception and performance, 24(2), 397.

Wohlschläger, A. (2001). Mental object rotation and the planning of hand movements. Perception \& Psychophysics, 63(4), 709-718.

Woolfolk, R.L., Murphy, S.M., Gottesfeld, D. \& Aitken, D. (1985a). Effects of Mental Rehearsal of Task Motor Activity and Mental Depiction of Task Outcome on Motor Skill Performance. Journal of Sport Psychology, 7, 191-197.

Woolfolk, R.L., Parrish, M.W. \& Murphy, S.M. (1985). The Effects of Positive and Negative Imagery on Motor Skill Performance. Cognitive Therapy and Research, 9, 3, 335-341.

Wulf, G. (2013). Attentional focus and motor learning: a review of 15 years. International Review of sport and exercise psychology, 6(1), 77-104.

Yue, G., \& Cole, K. J. (1992). Strength increases from the motor program: Comparison of training with maximal voluntary and imagined muscle contractions. Journal of Neurophysiology, 67, 1114-1123.

Ziessler, M., Nattkemper, D., \& Vogt, S. (2012). The activation of effect codes in response preparation: new evidence from an indirect priming paradigm. Frontiers in Psychology, 3, 585. 


\section{Footnotes}

1. The muscles that control finger movements are located in the arms, not the fingers. The particular sound when snapping the fingers is produced when the finger hits the palm of one's hand, not by the fingers rubbing together. One makes a bicycle go left or right by leaning in this direction, not by moving the handlebars. 